\title{
Die Überwindung des Religionsbegriffs in der Religionsphilosophie ${ }^{1}$ ).
}

Von Paul Tillielh, Berlin.

Es ist meine Pflicht, die Paradoxie in der Formulierung meines Themas za begrïnden. "Paradox" kann den Sinn von "geistreich" haben, dann beraht die Paradoxie auf der widersprachsvoll zweideutigen Wortform und gehört in die ästhetische Sphäre. Sie kann auch dialektisch sein. Dann beruht sie anf dem Zusammenstoß zweier widersprachsvoller, aber in sich notwendiger Gedankenreihen, und gehört in die logische Sphäre. In beiden Fällen liegt die Paradoxie im Subjekt, einmal in der Willkür der künstlerischen Phantasie, das anderemal in der Notwendigkeit-der logischen Konstitution. Nan aber gibt es einen Punkt, wo Paradoxie nicht im Sabjekt, sondern durchaus im Objekt begründet ist, wo Paradoxie zur Aussage ebenso notwendig géhört, wie Widerspruchslosigkeit zu jeder erfahrungswissenschaftlichen Aussage : Der Punkt, in dem das Unbedingte zum Objekt wird. Denn $\mathrm{daB}$ es das wird, ist ja eben die Erparadoxie, da es àls Unbedingtes seinem Wesen nach jenseits des Gegensatzes von Subjekt and Objekt steht. Paradoxie ist also die notwendige Form jeder Aussage über dạs Ưnbedingte. Die ästhetische wie die logische Paradoxie ist grundsätzlich auflösbar, beide stellen eine Aufgabe, sei es an den Witz, sei es an das Denken. Die Paradoxie des Unbedingten ist nicht auflösbar. Sie stellt eine Aufgabe an das Schanen. .

Das scheint die philosophische Aussage über das Unbedingte zư einer religiösen zu machen. Dazu ist zu bemerliken: Religionsphilosophie, die außerhalb der religiösen Wirklichkeit steht, ist so sinnwidrig, wie Ästhetik, die anßerhalb der künstlerischen Wirk-

1) Die folgenden Ausführungen stellen die Ausarbeitung eines in der Berliner Abteilung der Kant-Gesellschaft am 25. Jan. 1922 gehaltenen Vortrages dar. 
Paul Tillich, Die Überwindung des Religionsbegriffs usw. 447

lichkeit steht, denn beides hieße: Über einen Gegenstand reden, dessen einzige Gegebenheitsform unzagänglich bliebe. Dabei kann die Berïhrung mit der Sache die Form des schärfsten Gegensatzes annehmen, wenn dieser Gegensatz nur aus der Sacho selbist stammt. So hatte Nietżsche ein Recht, Gott zn bekämpfen, denn er tat es im Namen des Gottes, der durch ihn sprach; während Stranß kein Recht daza hatte, denn durch ihn sprach das Menschliche, allza Menschliche. Es ist daram sachlich begriindèt, wenn ich anf die geistige Gemeinschaft hinweise, in der ich mich in den folgenden Gedanken mit Männern des religiösen Wortes, wie Barth und Gogarten befinde. Es war überraschend für mich, za sehen, wie ohne gegenseitige Beeinflussung das nnbedingte "Ja" zum Unbedingten in dem religionsphilosophischen, wie in dem religiösen Denken za der prinzipiell gleichen Stelling geführt hät. Dennoeh sind die folgenden Gedankengänge gañz aus sich heraus zu. verstehen; sie sind Philosophie und sie sollen nichts sein als Philosophie. Die Paradoxie aller letzten Aussagen über das Ứnbedingte hindert nicht die Rationalität und Notwendigkeit der Begründungszasammenhänge, ans denen diese Paradoxie hervorwächst.

Es steht za beweisen, daß der Begriff der Religion in sich selbst eine Paradoxie enthält. „Religion" ist der Begriff einer. Sache, die eben durch diesen Begriff zerstört wird. Und doch ist er unvermeidlich; es käme also darauf an, ihn so zu verwenden, daß er einem höheren Begriff untergeordnet wird, der ihm seine zerstörende Kraft nimmt. Das aber ist der Begriff des Unbedingten. Es wird nun freilich infolge der inneren Dialektik des Religionsbegriffs eine gewisse $\mathrm{Z}$ weideutigkeit unvermeidlich sein, insofern das eine Mal der Begriff neutral, orientierend gebraucht wird, das andere Mal prägnant, polemisch. Dem ist nicht abzahelfen, denn jeder etwa nea geschaffene Begriff würde der gleichen Dialektik anheimfallen, der Zusammenhang maß entscheiden, was gemeint ist.

Wir sprechen I) Von dem Protest der Religion gegen den Religionsbegriff. II) Von der Herrschaft des Religionsbegriffs in der Religionsphilosophie. III) Von der Überwindnng des Religionsbegriffs. IV) Von der Dialektik der Autonomie. 


\section{I.}

\section{Der Protest der Rellgion' gegen den Religionsbegriff.}

Es sind vier Einwände, die die Religion gegen den Religionsbegriff erhebt. 1. Er macht die Gottesgewißheit relativ gegenüber der Ichgewißheit. 2. Er macht Gott relativ gegenüber der Welt. 3. Er macht die Religion relativ gegenüber der Knltur. 4. Er macht die Offenbarung relativ gegenüber der Religionsgeschichte. Insgesamt : Durch ihn wird das Unbedingte gegründet anf das Bedingte, es wird selbst bedingt, d. h. zerstört.

1. Die Gewißheit des Unbedingten ist anbedingt. Wo aber der Religionsbegriff das Denken leitet, soll es eine Gewißheit geben, die grundlegender ist als die des Unbedingten: - Die Gewißheit des Ich. Die Selbstgewißheit des Subjekts soll vor dèr Gottesgewißheit stehen. Vom Gottesbewaßtsein losgelöst soll das Iọh sich selbst erfassen. - Aber auch die Iochgewißheit ist kein Fundament unbedingter Gewißheit. Sie wird von einem Traumschleier über= deckt, wenn die Aaßenwelt, auf die sie bezogen ist, sich in Schein anflöst. Mit dem Objekt wankt auch das Subjekt. Das Unbedingte aber steht jenseits von Subjekt und Objekt. Nư wo dàs Ich als Stätte- der Selbsterfassung des Unbedíngten gemeint ist, nimmt es teil an der unbedingten Gewißheit, sei es des absolaten Lebens, wie beì Augustin, sei es der absoluten Form, wie bei Cartesius. Immer abẹr ist das Unbedingte das Begründende, das Ich das Medium und das Begründete. Wo es anders ist, wo das Ich sich loslöst, entsteht zwar - Religion, aber das İch verliert mit Gott zuletzt auch sich selbst.

2. Mit der Gewißheit des Unbedingten geht auch die Wirklichkeit des Unbedingten verloren. Die Religion raht als Funktion des Bedingten in der Welt des Bedingten. Und sie geht von dieser ihrer Welt ans, um zum Unbedingten zu gelangen. Sie hat einen selbstgenügsamen Weltbegriff, der nur an seinen Rändern einer Ergänzung bedarf. Und so wird Gott zu einem Korrelat der Welt, dadtrch aber selbst Welt. Und das wahre Unbedingte liegt jenseits von diesem Gott and der Welt. Es entsteht ein Gott unter Gott; der Gott des Deismus. - Oder der Weltbegriff bedarf keiner Ergänzung; das Universum ist in sich vollendet und Gott identisch mit ihm, die Totalität, die Synthesis aller endlichen Formen, das Universu $\vec{m}$ des Bedingten, das aber niemals das Unbedingte sein kann, der Gott des Panthëismus. - Wo der Welt- 
begriff ohne Gott fertig ist, da ist Gott ein bloßer Name, den man um der Religion willen ausspricht, den man aber auch weg: lassen kann, ganz gleich, ob das Universum Geist oder Materie genannt wird.

3. Der Geist des Religionsbegriffs vernichtet Gottes gewißheit und Gotteswirklichkeit, and er vernichtet die Religion selbst. "Religion" ist eine Funktion des menschlichen Geistes. Sie bleibt es auch, wenn man sie (mit Scholz) zu einer Schöpfung Gottes im Menschen macht. Denn zam mindesten muß der mensẹtliche Geist die funktionelle Möglichkeit zar Religion häbẹn, und mehr ist ja so wie so nicht gemeint. Sie steht also neben den übrigen Geistesfanktionen. Aber wo? Zuerst suchte sie ihre Heimat in einer anderen, der praktischen; aber die autonome Ethik ist fertig ohne sie, löst sie in sich auf oder schickt sie weiter; zu der theoretischen; aber diè autonome Philosophie braụcht sie nicht; stellt sie unter sich als Vorstafe, als Übërgang, löst sie in sich auf und schickt sie weiter, zum Gefühl; aber Gefühl begleitet jede Fünk= tion; also ein bestimmtes Gefühl; etwa fürr das Univierum; aber damit ist es nicht mehr die Funktion, sondern der Gegenstand̆, der die Religion bestimmt. Só wir̂̉ der Heimatlosen eine eigne Stätte gesucht, eine Provinz im Geistesleben (Schleiermacher) ein religiöses „Apriori“ (Tröltsch) die höchste' Aktklasse (Scheler) ; und so ist man ethisch, wissenschaftlich, ästhetisch, politisch und ist a a ch religiös. Das Unbedingte steht neben dem Bedingten; aber die Religion gestattet nicht, daß man a u ch religiös ist, sie gestattet überhaupt nicht, daß man "religiös" ist. Sie erträgt keine Nebenordnang, auch nicht in der Form einer Rangordnang; wo sie an erster Stelle steht. Sie ist ein verzehrendes Fener gegen alle autonomen Geistesfunktionen, and wer ein religiöses Apriori sucht, der muß wissen, daß damit alle anderen Apriori's im Abgrund versinken. Davon aber weiß der Religionsbegriff nichts.

4. Wie der Religionsbegriff die Unbedingtheit des Glaubens in die Relativität der Geistesfunktionen anflöst, so löst er die Unbedingtheit der Offenbarang in das Werden und Wandeln der Religions- and Kulturgeschichte anf; die Religion als Allgemeinbegriff ist indifferent gegen den Offenbarangsanspruch jeder Religion. Absolute Religion ist hölzernes Eisen; war das Christentum Religion geworden, so war es seiner Absolutheit a priori entkleidet. Und Tröltsch tat recht daran, das a posteriori festzn- 
stellen. Der Glanbe gibt das Prädikat "Religion" höchstens derjenigen Religion, die das Heil nicht bringt, der falschen Religion. Es ist ein herabsetzendes Wort und bezeichnet das Minderwertige in der Religion, dab sie im Subjekt stecken bleibt, daB sie lediglich Intention auf Gott hin ist, dab sie Gott nicht hat, weil Gott sich in ihr nicht gegeben hat. Und dieses Wort der Herabsetzung wird nun za dem Fundament, auf das die Offenbarang sich gründen soll - and doch nicht gründen kann. Denn entweder wird Offenbarang zar Mitteilung eines Wissens, das der autonome Geist auch sonst gefunden hätte. Sie löst sich auf in Rationalismus mit gelegentlicher Nachbilfe supranaturaler Art, oder sie wird Geistesgeschichte and löst sich auf in: die Bedingtheiten des Kaltarprozesses. Ist Offenbarung ein „religiöser ${ }^{\alpha}$ Begriff, so ist sie überhaupt kein Begriff mehr.

Das ist der Widerspruch der Religion gegen den Geist des Religionsbegriffs. Sehen wir zu, wie sich die bisherige Religionsphilosophie daza gestellt hat.

\section{II.}

Die Herrschaft des Religionsbegriffs in der Religionsphilosophie.

Die Religionsphilosophie ist im Abendland in drei Perioden verlaufen: die rationale, die kritische and die intuitive. Die empiristische Religionsphillosophie geht neben allen drei Perioden einher, kann aber hier außer Acht bleiben, da sie konsequenterweise nur über die Verwirklichang der Religion im seelischen und gesçhichtlichen Leben, nicht über sie selbst etwas ansșagen kann. Sobald sie es vénsucht, macht sie Anleihen bei einer der anderen Methọden.

1. Die rationale Periode ist die der unbewabten Herrschaft des Religionsbegriffes, die kritische die seiner bewnßten Herrschâft and die phänomenologische die seiner schwindenden Herrschaft. In der Philosophie der Renaissance ist das Weltbewaßtsein noch eingeschlossen in ein mystisches oder elsstatisches Gottẹsbewußtsein. Es gibt keine Welt abgesehen von Gott, wie es freilich in Unterschied rom Mittelalter keinen Gott abgesehen von der Welt gibt. Der Unterschied von Natar und Übernatur ist aufgehoben. Die Natar ist übernàtürlich; das' Übernatürliche Natür. Aber das war ein Übergang. - Die mathematisché Naturwissenschaft seit Galilei bannte das Übernatürliche. Die Natur wird rein gegenständlich, rein rational, rein technisch, sie wird außergöttlich. Es wird- 
Die Überwindung des Religionsbegrtffs in der Religionsphilosophie. $45 \frac{1}{2}$ möglich, einen Weltbegriff ohne Gottesbegriff za vollziehen. Damit aber ist der Herrschaft dẹs. Religionsbegriffs freie Bahn gèschaffen. Sie zeigt sich sofort am Ansgangspunkt der ganzen Entwicklung, bei Cartesius. Das Ich ist die Gewiłheitsgrandlage; vom Ich wird anf Gott geschlossen. Nicht das ist das. Verhängnis: volle, dab in der Selbstgewißheit des Ich das Prinzip aller Ratio: nalität gefanden wird: darin ist ja die Unbedingtheit der logischen Form enthalten, die als Unbedingtheit Heiligkêitsqualität in sich birgt. Aber daß nicht das Unbedingte daraus entnommen wird, um in ihm Gott $z \mathfrak{n}$ erfassen, sondern das Rationale, um mit ihina Gott za deduzieren, das zeigt die Veränderang der ganzen Liage, z. B. gegenüber Augustin. Sie kommt za voller Deutlichkeit erst in der Aufklärangsphilosophie, die mit Hilfe der téchnisch-gegenständlichen Kategorie Ursache und Zweck Gott aus der Welt erschließen will. Die Gottesgewißheit soll rahen auf' der Weltge: wißheit und der Kraft des logịschen Sehlusses. Das ist Herrschift des Religịosbegriffs; freilich in verhüllter Form, da überbaupt noch von Gott and nicht von Religion die Rede ist.

Kant hat richtig gesehen, daß ohne ontologischen Beweis dieses Ziel nnerreichbar ist. Aber der ontologische Weg war versperrt; er ist nur da möglich, wo das Bewabtsein in unmittelbarer Einheit mit dem Unbedingten steht, er ist dann kein logischer SchluB vom Denkẹn! anfs Sein des-Unbedingten, der natürlich unmöglich. ist, sondern er ist der Ausdruck für die unbedingte Gewißheit, die das Unbedingte allem Bedingten gegenüber hat, insofern es jenseits des Gegensatzes yon Denken and Sein steht. Mit' der Verselbständigung des Weltbewaßtseins, mit dem Auseinanderfallen von Denken and Sein, mit der Vergegenständlichung Gottes: wird dieser Ausdrack einer realen Bewaßtseinslage za einem Syllogismus, dessen Prämisse nicht zutrifft. So warde die Kritik des ontologischen Beweises das Fazit der Geistesentwicklung vom Mittelalter zur Neuzeit, vom Gottesbewabtsein zam antonomen Weltbewübtsein; and sie warde zugleich das Ende der rationalen Periode.

Der verhüllten Anfhebung der GottesgewiBheit entspricht die verhällte Aufhebung der Gotteswirklichkeit. Gott wird im Weltbild fast aller Philosophen dieser Periode das zentrale Moment der Weltkonstraktion: der Träger der Weltharmonîe, der geniale Uhrmacher des kosmischen Systems, der Vermittler von Subjekt and Objekt, immer technisch, immer gegenständlich, ein Ding anch wenn er der Ort der Ideen oder das Jenseits von Denker and 
Ausdehnung genannt wird. Denn auch das Denken ist durch den Determinismas der vorherbestimmten Harmonie dinghaft geworden. Der Gott, der die Welt ergänzen soll; ist Welt and nicht Gott. Allein Spinozas religiöse Tiefe überwindet diese Gottes unwürdigen Begriffe and weist in die folgende Periode; er selbst aber bleibt überwanden von dem dinghaften Weltbegriff seiner Zeit, durch den Gott gerade bei ihm zum absoluten Ding wird. Er enthüllt die Tendenz des Religionsbegriffs and mit Recht empfand ihn seine Zeit als ihre eigentliche Gefahr.

Auch die Unbedingtheit der Religion gegenüber der Kaltur ist in verhüllter Weise anfgehoben. Das ${ }_{n}$ colere et intelligere Deam" steht neben den "colere et intelligere" von Welt and Menschen. Wie Gott neben der Welt steht, steht die Religion neben Wissenschaft and Politik, neben Kanst and Sittlichkeit. Anch hier bleibt dịe zerstörende Konsequenz des Religionsbegriffs verhüllt. Man erkennt die Welt - and auch Gott; man hat den Staat - und auch die Kirche, man hat die Kunst - und auch den Kultus. Die Religion ist noch überall, aber sie ist überall ein Teil, and hat ihre Allgegenwart verloren. Dieselbe Verhüllang im vierten Pankt.- Die Absolutheit der Offenbarungswahrheit tritt auf als Absolutheit der Vernunftreligion. D.h. die Offenbarang ist ein Kapitel der Metaphysik geworden, hineinge-' zogen in die Dialektik des Widerlegens und Begründens. So lange trotz aller Widersprïche der Glanbe an die absolute Vernunft herrschte, blieb die Konsequenz des Religionsbegriffs verhüllt. Als die. Vernunft geschichtlich warde, ward die Vernunftreligion zur Religionsgeschichte.

2. In der kritischen Periode brechen die relativistischen Konsequenzen des Religionsbegriffs offen hervor. Die Gottesgewißheit verliert ihren theoretischen Sinn. Der moralische Gottesbeweis kanñ seinem wahren Gehalt nach nichts anderes leisten, als der sittlichen Autonomie die Weihe des Unbedingten zu geben: Alle Versuche aber, philosophischer und theologischer Kantianer, aus ihm mit ethischen Postalaten eine theoretische Existenz Gottes herauszaholen, sind vergeblich. Der Nenkantianismus hat darin die kláre Końsequenz der kritischen Grundlage gezogen. Und es ist das religionsphilosophische Verdienst der $„$ Philosophie des Als$\mathrm{Ob}^{\prime}$, diesen theoretisch existierenden Gott, der mit ethischen Postulaten erwiesen werden soll, als Fiktion durchschaut ,za haben. Für dỉe idealistischen Kantianer kommt eine Gottesgewißheit, ab- 
gesehen von der Weltgewißheit nicht in Frage. Die Religion ist eine besondere Art des Welterlebens, die' entweder in der Philosophie anfgehoben ist, wie bei Hegel oder eine danemde eigentütimliche Bedentang hat, wie bei Schleiermacher. Am deatlichsten ist die Wirkang des Religionsbegriffs da, wo nominalistisches Denken einen gegenständlichen Weltbegriff überhaupt nicht kennt, wie bei Simmel, und demgemäß die Religion ausschließlich ins Subjekt gelegt wird: die Religion ein Rythmus, eine Färbung der Seele, ein Ausdrack ihrer metaphysischen Bedeátsámkeit. Also eine Weihe nicht der gegenständlichen Welt, wie in Realismus, sondern des snbjektiven Lebens. Der Religionsbegriff, der vom Ich za Gott führen wollte, ist zam Ich zarückgesunken.

In der Fassung des Gottesgedankens senkt sich die drohende Wolke der vergangenen Periode, des Spinozismus nieder, seiner Dinghaftigkeit durch den idealistischen Ausgangspunkt entkleidet. Es gibt keinen Gott mehr abgesehen von der Wैelt. . Der Deismus wird zum Pantheismus. Gott ist die Weltidee, die Form der Formen, die letzte Synthesis, die als Realität oder unendliche Aufgabe gedacht wird; er ist die Welt sub specie aeternitatis. Dadarch ist wieder die Einheit von Gott und Welt hergestellt, aber nicht, wie in der Renaissance von Gott ans, der die Welt in sich anfgenommen, sondern von der Welt aus, die Gott in sich aufgenommen hat. Darum ist hier die gegenständlich-wissenschaftliche Begriffsbildang der Durchgang zu Gott. Der Weltbegriiff schafft. den Gottesbegriff and bält ihn in Abhängigkeit von sich. So ist es im Idealismas, so weiterhin: Der Gottesbegriff bleibt abhängig vom Weltbegriff: er geht mit ihm die materialistischen, voluntaristischen, naturalistischen, positivistischen Wege, also die Unerfüllbarkeit der romantischen Sehnsucht offenbarend, von der Weltform za Gott zn kommen, eine neue Unmittelbarkeit, eine nene ontologische Geisteslage von der wissenschaftlichen Welterfassung her zu erreichen. Und wieder ist es die "Philosophie des $\mathrm{Als}_{\mathrm{s}} \mathrm{Ob}^{a}$, die in klarer Erkenntnis der Sachlage die Entwurzelung durchschant hat, die den Gottesbegriff in dem Angenblick treffen muß, wo er za einer abgeleiteten Wirklichkeit herabgedrüekt ist, anstatt das Urgegebene selbst za sein.

Darans ergibt sich nar auch das Verhalten der kritischen Periode zu dem dritten Punkt: Entsprechend dem Pantheismus geht die Religion über in Kultur. Sie wird einer der Geistesfunktionen angehängt, and es bleibt nicht ans, dab sie sich in diese 
auflöst. Der Erfolg in der geistigen Lage des Jahrhanderts ist deutlich sichtbar: In einzelnen von Hegel abhängigen Denkern and in der von Hegel-Marx bestimmten Arbeiterscbaft nimmt die Wissenschaft die Stelte der Religion ein, in den ethisch-bürgerlich bestimmten Kreisen tritt die Moral in die Lücke, in den Schichten der höchsten Bildung die Kunst. Die Versuche, der Religion eine Sonderfanktion zu retten, miBlingen, weil ihre Absolutheit eine Relativisierung nicht verträgt, weil die geforderte religiöse Funktion genau so in Kultur umschlagen muh, wie der geforderte deistische Gott in Welt. Es ist freilich nicht zu verkennen, dab anf diese Weise die Kultur religiöse Weihe erhält; aber diese Färbung erhält sie nachträglich; sie kann auch fehlen und fehlt, sobald der Weltbegriff ans idealistischen in materialistische und voluntaristische Fassungen übergegangen ist.

Der Sieg der historischen Vernunft im Idealismas bedeatet auch den Sieg der Religionsgeschichte in dieser Periode. Sie war, durchaus als Offenbarungsgeschichte gemeint, natürlich nicht im supranaturalen, aber im immanent-geistesgeschichtlichen Șinn; es ist Gott selbst, der in ihr zum Selbștbewühtsein im Endlichen kommt; es sind die Weltpotenzen, die der Reihe nach in der Mythologie und Offenbarung sich kundgeben, - Mit dem Zerbrechen der idealistischen Voraussetzung wird die Offenbarungsgeschiehte ein Stück menschlicher Geistesgeschichte, dessen Sinn es istu, sich in Kultur= geschichte aufzulösen. Auch hier der völlige Sieg des Religionsbegriffes. - Die kritische Periode ist kensequenter als die rationale; das ist ihr Voržng; sie enthüllt die religionszerstörenden Folgen des Religionsbegriffes, aber sie leistet auch etwas Positives. Sie ist eine machtvolle Reaktion gegen die gegensiständliche Ent= leerung and Entheiligung der Welt. Diese Reaktion bleibt zwar romantisch and ästhetisch and schlägt wieder in ihr Gegenteil um; denn das zerstörte religiöse Bewuß̉tsein kạnn nicht durch.Wille einzelner, sondern nụr,durch Schicksale von. Völkern und Massen w̌iedërge w̌onnen werden. "Aber die romantische Religionsphilosophie gibt dennoch die Brïcke und schafft Formen, über die wièder der neñe Geíst ontölogischen Góttesbewuß̣tseins sich ergießen könntẹ.

Welt, Kaltur, Geschichte hạbẹn Heiligkeitsqualitäten, können sie haben, aber brauchen sie nicht za haben. Wie aber, wenn die Ördnung umgekehrt würde; wenn es hieße: Müssẹn sie haben: wenn vor allem dạs Religiöse. Unbedingtheít und Gewißheit hätte and 
die Welt and die Kaltur und die Geschichte zeitliche, zweifelhafte, zu überwindende Profanisierungen des Heiligen wären? Miț dieser Frage wenden wir uns der dritten, intuitiven Periode ża.

3. Sie beginnt mit der Jahrhandertwende; nicht nuir durch die im engeren Sinne phänomenologische Philosophie, sondern durch die allgemeine Bewegung des Geisteslebens hinweg von der gegenständlich-technischen zu einer urständlich-intritiven Welterfassung. Es ist schwerer über sie etwas zu sagen, da sie erst in Entfaltung begriffen ist; aber es ist doch schon möglich, sie in den weitesten Umrissen za erschanen. Für die Religionsphilosophie hat sie die Bedeutung, gegen die Herrsehaft des Religionsbegriffs bewuBt anzugehen. Es scheint sich eine neue ontologische Geisteslage anzubahnen. Die Erfassung des Numinösen durch Otto als einer alle Gegenstandsformen darchbrechenden Wirklichkeit, die Erhebung des Heiligkeitswertes über die ụ̈brigen Wertstufien durch Scheler, die völlige Trennang des religiösen von dem theoretischen Existentialurteil durch Scholz liegen in' dieser. Richtang. Wir stellen nun die Frage, wieweit gelingt es hier, den Geist dệs Rèligionsbegriffs zu bannen?

Scheler wie Scholz wollen die fanktionelle Begründang 'der Religion mit Energie überwinden, Scheler, indem er dem religiösen Objekt die primäre Gewißheit gegenüber dem religiösen Akt za schreibt und die Gottesfrage vor der Religionsfrage erledigt, Scholz, indem er die Auffassung der Religion als antonomer Geistesschöpfung bestreitet and in dem Satze "Gott ist" das erste. Wesensmerkmal der Religion sieht., Es könnte eingewandt werden, dab damit eine Wiederkehr der rationalen Methode droht; aber die Gefahr besteht in Wirklichkeit nicht. Nicht mit Hülfe von Syllogismen soll aus einem feststehenden Weltbegriff Gott erschlossen werden, sondern ohne Berücksichtigung der Welt soll seine Wirklichkeit erschaut werden. - Um die Scheidung dieses Anschanens von der reflektiv-gegenständlichen Welterkenntnis hervorzaheben, bant Scheler die Wirklichkeitserfassung in Stufen auf: die wissenschaftliche, die metaphysische und die religiöse Erkenntnis. Zweifellos ist damit eine Überwindung sowohl der rationalen wie der kritischen Methode angebahnt. Aber doch nicht erreicht. Denn es ist nicht dentlich, wie sich die Stafen za einander verhalten. Welche Schwierigkeiten hier vorliegen, zeigt Scheler, wenn er die Metaphysik mit einem sacrificium intellectus sich selbst aufheben läßt, za Gansten der Religion. Damit ist das Gottesbewabitsein ab- 
hängig gemacht von einem sich selbst vernichtenden WeltbewaBtsein; die Gottesgewißheit lebt vom Opfer der WeltgewiBheit; die Gotteswirklichkeit vom Opfer der Weltwirklichkeit. Aber dieses Opfer bringt die Welt. Gott lebt vom Opfer und er schwindet, wenn der autonome Geist das Opfer weigert. Der aber muB es weigern, um nicht durch theoretische. Urteile, die fremder Quelleentspringen, in sich zweispältig za werden.

Der protestantische Religionsphilosoph Scholz fordert nicht ein sacrificium intellectus, sondern er sucht dem Intellect die Glanbwürdigkeit der Religion za beweisen. Er setzt also ein Bewußtsein vorans, für das die Glanbwürdigkeit bewiesen werden müßte. Dieses Bewaßtsein aber ist dàs der sittlichen Persönlichkeit. An der ethischen Qualität der Offenbarungsträger hat sich das Vertrauen anf die Wahrheit ihrer Offenbaring zu entziüinden. Wer sieht hier nicht den ins Persönliche transponierten moralischen Gottesbeweis, der dem Protestantismus so tief im Blat sitzt? In beiden Fällen ist die Weltgewißheit and die Weltwirklichkeit als das Grundlegende beibehalten gegen̈über der Gottesgewißheit und Gotteswirklichkeit, eînmal âls Stufe, das andere Mal als K Kriterinum. Erreicht ist nur ein doppeltes. 'Gọtt wird weder erschlossen, wie in der rationalen, noch in die Welt hineingẹzogen, wie in der kritischen Periode.

Auch in den beiden anderen Pankten; Religion und Kultur, Offenbarang and Geschichte, hilft sich ScheIer darch den Stafengedanken: die religiösen Werte sind die höchsten in der Wertrèihe; Heiligkeitswërte stêhen noch über Persönlichkeitswerten. Und innerhalb der Heiligkeitswerte .steht wieder die im Christus gegebene Gotteswirklichkeit an erster Stelle über Propheten und Heiligen: die Religion der höchste Kultạrwert, die christliche Religion der höchste Heiligkeitswert. - Offenbar herrscht anch hier noçh der Religionsbegriff. Die Stufenreihe läßt die höhere Stufe auf die niederen gegründet sein im Sinne des Bildes, wie im Sinne der Sache, die es veranschanlichen soll; es bleibt ein Denken vọn untèn, ein Êmporstéigen; aber ès gibt keine Stufen, die zự Unbedingten führen; die höchste: wịe die niedrigste ist von dèm Unbedingten gleich weit entfernt.

Bei Scholy tritt aüch hier ân Stelle der Stufenlehre, deren katholisch-mittelalterlicher Ursprung ja deutlich ist, die ethischkultarelle Pers̈önliçhkeitsidee, deren protestantische Wurżel offen liegt: die Religion ist eine dem ̈̈brigen Geisteslében gegenüber 
selbständige Sache, die da sein, aber aneh fehlen kann; ist sie aber da, so ist das Maß ibrer Wertung die Erlebbarkeit durch den Kultarmenschen der Gegenwart, d. h. durch die geistig-ethisch geformte Persönlichkeit. An erlebbaren Religionen aber kommen schlieblich nur drei in Betracht; das Christentum, der Pantheismus, die Mystik. - Es widerspricht nün schlechterdings der Unbedingtheit des Unbedingten, daB es in seiner Art und seinem MaB abhängig gemacht wird von dem $\mathrm{Ma} \beta$ einer bestimmten geistig-ethischen Persönlichkeits - oder Kulturlage. All diese Gedanken entstammen noch einem Denken, das nicht auf das Unbedingte, sondern Bedingte sieht, um an ihm das Unbedingte za messen. Sie haben den Geist des Religionsbegriffes nicht bannen können. Aber-ist er überhaupt zu bannen? Oder ist es das Verhängnis der Religionsphilosophie, ihm verfallen za sein? Ist es das Ver... hängnis der menschlichen Geschichte, daß es nur eines̃ in.ihr geben kann, Religion oder Religionsphilosophie?

\section{III.}

\section{Die Überwindung des Religionsbegriffs.}

Der entscheidende Einwand, den wir gegen die bisherige Religionsphilosophie erhoben, ist $\mathrm{der}, \cdot \mathrm{daB}$ sie das Unbedingte auf das Bedingte gründet entweder durch Nebenordnung oder, da diese nnerträglich ist, durch Auflösung des Unbedingten in das Bedingte. Eine Religionsphilosophie, die dem Weșen des Unbedingten gerecht werden will, muß das Unbedingte in allem Bedingten erfasssen, als das, was sich selbst and dás Bedingte begründet. Das Bedingte ist das Medium, in dem und darch das hindurch das Unbedingte erfabt wird. Zu diesem Medium gehört anch das erkennende Subjekt. Auch dieses tritt in keiner Weise als begründend auf, sondern nur als der Ort, in dem das Unbedingte im Bedingten offenbar wird. Darans folgt, daß der Sinn jeder Aussage über das Unbedingte prinzipiell anterschieden sein maß von dem Sinn jeder Aussage über Bedingtes. Da aber jede Aussage als solche in dem Schema von Sabjekt und Objekt, also in den Formen des Bedingten verläuft, so maß die Aussage über das Unbedingte diese Formen zwar benatzen, aber doch so, daB ihr Unzalängliches offenbar wird, d. h. sie mab die Form der systematischen Paradoxie tragen.

1. Die Selbstgewißheit des Ich ist unter der Herrschaft des Religionsbegriffs begründend für die Gottesgewilhheit. Non aber ist in der SelbstgewiBheit des Ich ein Doppeltes enthalten: Das 
Unbedingte einer Realitätserfassung, die jenseits von Subjekt and Objekt liegt und das Teilhaben des subjektiven Ich an diesem Unbedingt-Wirklichen, anf dem es raht. Das Ich ist das Medium der unbedingten Realitätserfassung; und es nimmt als Medium teil an der Gewißheit dessen, was es vermittelt; aber es nimmt. nar als Medium teil; es ist nicht das Tragende, sondern das Getragene. - Es besteht nan für das Ich die Möglichkeit, seine Selbstgewißheit so za erleben, daß die unbedingte Realitätsbeziehung, die darin enthalten ist, im Vordergrund steht: die a priori religiöse Art der Selbsterfassung; es besteht andererseits die Möglichkeit, seine Selbstgewißheit so zu erleben, daß die Beziehang auf das Sein des Ich im Vordergrund steht, diè a priori unreligiöse Art der Selbsterfassung; im ersten Fall dringt das Ich gleichsam durch die Form seiner Bewußtheit hindurch, za dem Realitätsgrund, auf dem es ruht, im zweiten Fall bleibt dieser Untergrund zwar wirksam - ohne ihn gäbe es keine Selbstgewißheit - aber er wird nicht angetastet; das Ich bleibt in seiner Losgelöstheit, in der Bewûßtseinsform. Kann man diese zweite Stellung auch mit Recht unreligïös nennen, so doch nar, insofern die Intention in Betracht kommmt, nịcht sowẹit es sich um den Erfolg handelt. Ein der Substanz nach unreligiöses Bewưtsein gibt es nicht, wohl áber der Intention nach. In jeder Ich-Erfassung ist die Beziehung auf das Unbedingte als Realitätśgründ enthalteñ; aber nicht, in -jeder ist sie gemeint; danach unterscheiden sich die beiden Lagen deś Bewaßtseins.

Die Aussage; daß in der Selbstgewißheit die Gewißheit des Unbedingten erfaß̧t wird, ist päradox; denn sie hat die Form des. Theoretischen and ist doeh dem Theoretischen schlechterdings fremä. Wenn gesagt wird; dab Ich erfasse in sich das Unbedingte als Grund seiner Selbstgewißheit, so ist in der Form dieser Aussage der Gegensatz von Subjekt und Objekt enthalten; aber der Gehailt dieser Anssage steht dem gerade entgegen: das Unbedingte ist́ nicht Objekt, es ist auch nicht Subjekt, sonderñ es ist die Voraussetzung jedes möglichen Gegensatzes von Subjekt and Objekt. Darum steht die Erfassung des Unbedingten auch vor jedem theoretischen Ürteil. Und ist in Grund 'und Folgen unabhängig von aller theoretischen Gewißheit. Ob der Geist die religiöse oder unreligiöse Intention in sich trägt, - ist theoretisch , jndifferent, da das Unbedingte zwar das Tragende "auch alles theoretischen Urteills ist, selbst als absolute Voraussetzang aber niemals Gegenstand 
der Theorie sein kann. Wird es das doch - and es mub es jà werden, da sonst überhaupt nichts ausgesagt werden könnte - so hat diese Aussage notwendig paradoxe Form: Gottesgewißheit ist die in der Selbstgewißheit des Ich enthaltene und sie begründende Gewißheit des Unbedingten. Damit ist die Gottesgewißheit schlechterdings unabhängig von jeder anderen vorausgesetzten Gewißheit. Das Ich and seine Religion steht unter dem Unbedingten; es ist erst möglich durch das Unbedingte. Es gibt deswegen überhatpt keine GewiBheit, in der nicht die Gottesgew̄ißheit implicite enthalten wäre; aber ob sie auch explicite enthalten ist, das macht den entscheidenden religiösen Unterschied aus. Objektiv ist jẹdes Bewabtsein Gott-gebanden, aber subjektiv laann das BewaBtein Gott-los sein. Es gibt also keinen Weg vom Ich zu Gott; aber es gibt - der Richtung, nicht der Substañz nach - éinen Weg von Gott weg zam Ich. Ist dieser Weg einmal béschritten, so gibt es auf ihm freilich kein Zarück: Nür der Dürchbruch des im IchBewaßtsein enthaltenen Grundes durch die antonome BewaBtseinsform befreit von dem Zwang der Gottesferne; die Religion nennt diesen Darchbrach Gnade. Sie weiß, daß kein theoretisches Hinweisen auf das aller Theorie za Grunde Liegende das Unbedingte im BewaBtsein lebendig machen kann; denn die Theorie hat das Unbedingte als Objekt, also als das, was es nicht ist.

2. Die Weltwirklichkeit begründet unter der Herrschaft des Religionsbegriffs die Gotteswirklichkeit. Nun steht jedes Wirkliche in den Formen der Gegenständlichkeit, zu denen auch die Existenz gehört; zugleich aber ist darch jedes Wirkliche erfaßbar ein Unbedingt-Wirkliches, das nicht in den Formen der Gegenstände steht, also auch keine Existenz hat. Wo der Geist sieh so auf die Welt and ihren Inhalt richtet, daB er das Moment der Unbedingtheit, das in allem enthalten ist, ins Bewabtsein erhebt, da ist er anf Gott gerichtet. Dieses Moment der. unbedingten Wirklichkeit in allem Bedingt-Wirklichen, ist das, was tragend ist in jedem Ding; es ist seine Seienswarzel, seine Ernsthaftigkeit, seine Unergründlichkeit, seine Heiligkeit. Es ist sein Realitätsgehalt im Unterschied von seiner zufälligen Form.

Jedes gegenständliche Denken ist hier streng auszuschließen: Es ist nicht von einem Gegenstand neben den Dingen, oder über den Dingen oder in den Dingen die Rede; es ist überhanpt von keinem Gegenständlichen, sondern von dem Urständlichen. schlechthin die Rede, dem was aller Form, auch der Existenz enthoben 
ist. Aber auch hier gilt, daß jede Aussage gegenständliche Form hat, and darum nur als gebrochene, paradoxe Aussage wahr ist.

So ist die Aussage "Gott ist" der Form nach eine theoretische Aussage and keine Stufenordnang kann das ändern; es ist die Einreihnng Gottes in die Gegenstandswelt; aber diese Einordnung ist Gottlosigkeit.. Ist die Aussage "Gott ist" auch dem Gehalt nach theoretisch, so vernichtet sie die Gottheit Gottes. Ist sie aber als Paradoxie gemeint, so ist der notwendige Ausdruck für die Bejahung des Unbedingten; denn es ist nicht möglich, sich anders auf das Unbedingte zu richten als darch Vergegenständlichkeit. - Damit ist Deismus und Pantheismus überwanden. Der Deismus, der nicht nur eine Zeit-Richtung, sondern ein Element ist in jeder Gottesvorstellang, das Moment der Vergegenständlichung. Verendlichung Gottes, das überall auftritt, wo der paradoxe Sinn des göttlichen Seins nicht mehr erfaßt wird; und des Pantheismas, der das Unbedingte mit der aniversalen Dingform, der Welt, gleichsetzt, weil das Unbedingte durch jedes Wirkliche hindurch erfaßbar ist, der aber dann doch bei einer Gegenstandsform, der universaleñ, stehen bleibt, and nicht sieht, daß dàs Unbedingte der Totalität so fern ist, wie der-Einzelheit. Es ist Platz für einen Theismus, der nichts gemein hat mit dem üblichen kirchlichen Semi-Deismus, sondern der nür sagt, daß das Unnbedingte - das Unbedingte ist.

Auch für diese Haltung gibt es keine theoṛetische Notwendigkeit.. Es ist möglich, sich auf das System des Bedingten zu richten und és in seiner Selbstheit zu bejahen, wie das autonome Ičh. Es ist möglich, sich von der Bẹziehung auf dàs UnbedingtWirkliche, dàs allem innewohnt,-abzuẉenden zu der Existenz und der Form des Gegenständlichen; denn jedes Ding in der Welt hat die Form der Existenz und des Objektiven. Es ist das möglich ohne theoretische Bedenken, denn das Unbedingte ist nie und nirgends ein theoretisches Streitobjekt; man kann von der Theorie weder dafüir'noch dagegen Partei nehmen; es begibt sich nicht in die' Kampfarena der Existential-Urteile, der Fragen nach Dasein oder Nichtsein. Ist man aber einmal unter Verzicht auf die Gotteswirklịchkeit zn einer Weltwirklichkeit gekommen, die der Absicht nach - der Substanz nach ist es es unmöglich - außergöttlich ist, so gibt es keinen Weg zar Gotteswirlzlichkeit zurück. Denn Gott ist entweder der Anfang oder er ist. nicht. 
3. Die Religion wird unter der Herrschaft des Religionsbegriffs aus der Kultur begründet, entweder als einzelne Kultarfunktion. oder als Synthesis der Kalturfunktionen. Das ist durchaus analog der deistischen und pantheistischen 'Gottesauffassung. ' Nun gibt es aber eine Funktion des Geistes, die weder neben dem andèrn steht noch ihre Einheit ist, sondern in ihnen und durch sie hindurch züm Ausdruck kommt: die Funktion der Unbedingtheit; sie ist die Wurzelfanktion, diejenige, in der der Geist durch alle seine Formen hindurchbricht bis auf , seinen Grund. Sie ist deswegen auch keine Geistesform und kann nur durch Paradoxie Funktion genannt werden. Phänomenologisch gesprochen: es gib̆t eine Aktklasse, die aus einer Tiefe stammt, in welcher der Gegensatz von Akt zu Akt aufgehoben ist, und die infolgedessen nar dareli Brechung im Medium des Bewaßtseins zu eigenen Akten kommen kann. Ihrem Wesen nach aber ist sie nichts anderes als die Beziehang auf das Unbedingte, die jedem Alit Innewohnt. - Es gibt also keine besondere religiöse Funktion neben der logischen, ästhetischen, ethischen, sozialen; sie ist auch nicht in einer oder in der Einheit aller enthalten, sondern sie ist der Durchbrach darch. jede und die Realität, die unbedingte Bedeutung einer jeden. Die Kaltar ist das Medium des Unbedingten im Geistesleben, wie die Dinge das Medium des Unbedingten in der Welt sind.

Damit ist anfs Nachdrücklichste bestritten, dab darch die Religion ein neuer Wert in das System der Werte eingefiuhrt ist. Es gibt keine Heiligkeitswerte, sondern das Heilige ist das, was den Werten den Wert gibt', die Unbedingtheit ihres Geltens, die Absolutheit ihrer Realitätsbeziehung. - Es ist. die Religionsphilosophie also nie und nimmer eine Ergänzung der Geistes- oder Wertphilosophie. Auch an diesem Punkte tritt das. Unbedingte nicht in die Diskassion der Bedingtheiten. Die Heiligkeitsqualität, die Unbedingtheitsfunktion kann fehlen, ohne daß das System der Werte im Mindesten verändert wird; sie kann fehlen, freilich nar der Intention, auch hier wie überall - nicht der Substanz nach; denn fehlte sie, wäre das Denken wahrheitsand das Anschanen wesenlos, das Handeln ziel- und die Gemeinschaft seelenlos. Aber sie braucht nicht gemeint za sein. Der Geist kann sich richten auf die Antonomie seiner Funktionen, deren Realitätswarzel er nicht anrührt, deren Form er durchsetzt. Der Geist kann antonome Kaltur schaffen, mit einem autonomen Ich, in einem autonomen Universum. Damit aber hat er sich den 
Weg zu Gott versperrt. Auf dem Boden der autonomen Kultur gibt es höchstens - Religion.

Hier ist nun der Ort, die Dialektik des Religionsbegriffs zu völliger Durchsichtigkeit zu bringen: Sobald das Bewußtsein sich auf das Unbedingte richtet, entsteht die Doppelheit von Akt and Gegenstand. Nan ist der religiöse Akt aber kein besonderer; er ist nar in den übrigen Akten wirklich. $\operatorname{Er} m a B$ diesen also eine Formung geben, an der die religiöse Qualität sichtbar ist. Diese Formung ist die Parodoxie, d.h. zagleich die Bejahang and Verneinung der autonomen Form. Das religiöse Denken, Anschauen ist also ein Denken, ein Anschanen, das die autonomen Formen des Denkens und Anschauens zagleich benutzt and zerbricht. Das Gleiche gilt von den sittlichen und sozialen Formen.

Das Erkennen unter der Gegenwart des Unbedingten ist Inspiration. Das Anschanen ist Mysterium, das Handeln Gnade, die Gemeinschaft Reich Gottes. Alles das sind paradoxe Begriffe, d.h. solche, die sofort ihren Sinn verlieren, wenn sie gegenständlich gemacht werden; Inspiration als eine übernatürliche Art der Erkenntnisvermittlung ist ein einfacher Widersprach. Mysterium im Sinne einer materiell-realen Gegenwart, das Unbedingte im Bedịngten ist eine sinnllose Aussage; die Gnade als übernatürliche Kraftmitteilung ist ein ethischer Nonsens and das Reich Gottes als reale Grọ̈e eine Utopie mẹchanistischen Denkenș. An Stelle des Parodox ist der Supranaturalismus getreten; d.h. der Versuch, ein Bedingtes anbedingt zu machen. Dem Supranaturalismus äber entspricht immer der Nataralismus, d. h. der Versuch, das Unbedingte überhaupt auszaschalten.

'Und doch kann die Religión nicht anders; als mit diesen Begạffeñ arbeiten; sie mụß vergegenständlichen, um anssagen za können; dąi sie aussagen will, ist ihre \#leiligkeit; daß sie gegenständlich aussagen mub, ist ihre Profanheit: Trerechtfertigt ist sie nur da, wo sie diese ihre Dialektik durchschar and dem Unbedingten allein die Ehre gibt. - Wo sie es ñicht tut, führt sie das Unbedingte in die Niederang and die Kampfarena des Bedingten herab, in der es notwendig unterliegen muß: Es wird eine Kultur, die die Beziehung auf das Unbedingte verloren hat, ein Denken, das nichts mehr weiß von Inspiration als dem Durch'bruch der unbedingten Realität, ein Anschanen, das nichts-mehr weiß vom Mysterium des Grundes in den Formen der'Dinge, ein Hăndeln, das ohne Gnade dem Gesetz verfallen ist, einè Gemein-· 
Die Überwindung des Religionsbegriffs in der Religionsphilosophie. 463

schaft, die fern ist von dem Burchbrechen der unbedingten Liebe - das auf der einen Seite; und eine Religion, die aus all diesen Begriffen supranaturale Gesetze, Objëktivierungen der Paradoxie, Verendlichangen des Unbedingten gemacht hat: das ist der Zastand des Geistes unter der Herrschaft des Religionsbegriff's: -Erlösang der Religion vom Verhängnis der Objektivierung, Erlösung der Kultar vom Verhängnis der Profanisierung, Darçh̆bruch des Unbedingten darch alle Arten der Relativisiernng, das ist Sieg über den Geist des Religionsbegriffs.

4. Unter der Herrschaft des Religionsbegriffs gründet sich die Offenbarung anf das autonome Geistesleben, sei es im Sinne einer. offenbarten Vernunftreligion, sei es im Sinne der Religionsgeschichte. Dadurch wird die absolute Tat Gottes zu einer relativen Entwicklnng des religiösen Geistes. Die Religion ạber will nicht Religion, auch nicht absolute Religion, sondern sie will Er. lösüng, Offenbarang, Heil, Wiedergeburt, Treben, Vọllendung, sie will das unbedingt Reale, sie will Gott. Und sie nennt währe Religion die, in welcher Gott sich gibt, und falsche die, in welcher er vergeblich gesucht wird. - Der Religionsbegriff aber kann derartige Unterschiede nicht anerkennen, auch nicht in der verhüllten Form von erlebbarer and nichterlebbarer Religion. Der Religionsbegriff macht gleich, bringt Göttliches and Menschliches anf eine Ebene. - Nun aber ist es selbst schon eine Wirkang des Religionsbegriffs, selbst schon eine Bedingtmachung des Unbedingten, wenn eine bestimmte Religion unbedingt gesetzt, mit der göttlichen Offenbarung gleich géstellt wird. Jede Religion ist als Religion relativ, denn jede Religion ist Vergegenständlichung des Unbedingten. Aber jede Religion kann als Offenbarung absolut sein; denn Offenbarung ist das Darchbrechen des Unbedingten in seiner Unbedingtheit. Jede Religion ist insoweit absolut, als sie Offenbarung ist, d.h. insoweit als das Unbedingte in ihr als Unbedingtes herantritt im Gegensatz zu allem Relativen, was ihr als Religion zakommt.

Es ist non aber die Eigenschaft jeder lebendigen Religion, dab sie eine ständige Opposition gegen das Religiöse in ihr in sich trägt. Der Protest gegen die Vergegenständlichung ist der Pulsschlag der Religion. Erst wo er fehlt, ist nichts Absolutes mehr in ihr, ist sie ganz Religion, ganz Menschliches geworden. - Es sind aber drei Formen, in denen sich der typische-Protest der lebendigen Religion gegen ihre Vergegenständliohang als Re- 
ligion erhebt: die Mystik, die Prädestination, die Gnade. Die Mystik durchschaut den paradoxen Sinn aller Aussagen iuber das Unbedingte. Sie sucht die Einheit mit dem absolut Gegenständlichen, dem Abgrund, dem Überseienden, dem reinen "Nichts". Sie weiß anch, daß diese Einheit nur vom Unbedingten her geschaffen werden kann, sie weiß, daßB sie Gnade ist. - Aber ssie bereitet sich doch vor, der Gnade würdig zu werden and sie benutzt daza die Formen der Religion und schafft selbst Formen. Sie verläßt den Boden der Religion nicht. Das ist ihre Grenze. - Die Prädestination überläbt alles Handeln zum Heil des Einzelnèn and der Menschheit Gott. Weder Kirche noch Religion sind Bedingungen der Erwählung und des Reiches Gottes, sie sind höchistens ihre Grott-geordnete Vermittlung; dadurch sinkt ihre Bedeutang dahin und da der göttliche Ratschlaß im Verborgenen geschieht, so ist alles religiöse Handeln and Vorstellen des Menschen entwertet, and kommt bald dem Pankt nahe, wo es ganz aufhört und übergeht in profanes, kultarelles Handeln; das ist die Gefahr, wenn das Religíöse ganz ins Verborgene and Absolnte gestellt wird. - Die konkrete Gnade (von Gnade lebt ja anch Mystik und Prädestination) stellt das Heil gleichfalls schlechterdings in das Ứnbedingte; aber nicht in seinen Abgrand and nicht in seinen-verborgenen Willen, sondern in seine konkrete geschichtliche Selbsstmitteilung. Es fälltt von hier aus ein 'starkes Ja anf die kirchlich-religiösen Medien, auf Offenbarangsmittler und Offenbarangsmittel, auf Gebet und lebendige Gemeinschaft mit Gott, Hier ist der Abweg fast unvermèidlich, daß diese Medien ins Absolute'erhoben und aus der Offenbarung der Gnade Rèligion der Gnadenmittel wird.

Jede der drei Formen, in denen innerhalb der Religion die Religion überwanden wird, haben aIso die gleiche Dialektik wie die Religion selbst, sie können sich an Stelle Gottes setzen. 'Es ist deswegen auch falsch, diese. Formen zur absoluten Religion zu machen, Sie sind Ausdrucksformen für das àbsolate. Element jeder lebendigen Religion, aber sie werden selbst relativ, sọbald sie Religionsformen werden: Die absolute Religion geht durch alle Religionèn hindurch; die wahre Religion ist überall da, wo das Unb̧edingte als Ünbedingtes bejaht und die Religiọn vor ịm vernichtet wird.

Wo das. geschieht, ist im Allgemeinen verborgen. Offenbar wird es dann und wann in Form der großen mystisehen oder pro- 
Die Überwindung des Religionsbegriffs in der Religionsphilosophie. 465

phetischen Reaktionen gegen die blobe Religion. Das Maß, in dem eine Religion zu solchen Reaktionen fähig ist, entscheidet über ihren relativen Rang. Absolute Religion ist niemals ein gegenständliches Faktum, sondern ein jeweils lebendiger Dürchbräch des Unbedingten. Den Beweis der Absolutheit führt Gott selbst, indem er den Absolutheitsanspruch einer Religion zerbricht, nicht darch Skepsis and Religionsgeschichte, sondern durch die Offenbarung seiner Unbedingtheit, vor der alle Religion nichts ist.

Es ist also auch hier das Unbedingte das Tragende, das Fandeln Gottes die Sabstanz der Religion, ohne die sie nicht sein kann; aber sie kann sich von ihr abwenden; sie kann mit and ohne BewaBtsein diese Substanz unangerührt lasssen and sich ihrer eigenen autonomen Form zuwenden. Sie kann autonome, selbstgenügsame, Gott-ferne Religion werden, and den Grötzendienst dadurch vollenden, daß sie sich absolute Religion nennt.

Damit sind die vier Vorwürfe der Religion gègen die Religionsphilosophie in ihrem Rechte anerkannt; aber es ist nicht die Folgerang daraus gezogen, daß ự der Religion : willen die Relígionsphilosophie sich selbstt aufgeben mïßte, sondern es ist der Versuch gemacht, eine Religionsphilosophie auf die Forderungen zn gründen, die in jenen Vorwürfen enthalten sind, d: h. eine Religionsphilosophie; die nicht vom Bedingten, sondern vom Unbedingten, die nicht von der Religion, sondern von Gott ausgeht. An dem Gelingen oder Mißlingen nieht dieses meines, aber eines solchen Versuches überhaupt hängt das Schicksal der Religionsphilosophie and damit der Stellang des Geisteslebens zan Religion. Wir stehen vor der Alternative: Entweder Aufhebung der Religion durch die Kultur, oder Durchbrechen des Unbedingt-Wirklichen als des Grundes oder der Realität aller Kaltur in all ihren Fnnktionen. Die Art, wie innerhalb der Wissenschaft sich dieser Durchbruch vollziehen könnte, sollten die ausgesprochenen Gedanken andeaten. Was das Zilel betrifft, so kann es für mich keinen Zweifel geben; was die Form betrifft, so ist sie ein Versuch, and nicht mehr.

IV.

\section{Die Dialektik der Autonomie.}

Alles Gesagte hat im Grande das Ziel, einer Bewabtseinslage den Weg za bereiten, in der die Selbstgewißheit des Bedingten zerbrochen ist vor der Gewibheit and Wirklichkeit 
des Unbedingten. Nicht die Lösnng eines theoretischen Problems war mir die Hauptsache, sondern die Anfweisung einer Geisteslage, anf die meiner Ûberzeugang nach -schicksalsmäBig die Geistesbewegung hindrängt. Um so mehr ist es meine Pflicht, Rechenschaft za geben über die Denkmittel, die zar Anwendang gekommen sind. Es ist aber ein Doppeltes, was dabei heranszastellen ist, eine bestimmte Methode and eine bestimmte Geschichtsphilosophie, eine logische and eine metaphysische Voraussetzang.

1. Die Methode, die am schärfsten in der Analyse der Selbstgewißheit, aber auch an den anderen Punkten zar Anwendung gebracht ist, kann als kritisch-intuitive Methode angesprochen werden. Sie geht davon ans, dab sowohl die kritische, wie die intuitive Methode in Absonderung unfähig zur Lösung des religions- and damit kulturphilosophischen Zentralproblems ist: der Frage nach dem Sinn oder besser der Realität, der unbedingten Ernsthaftigkeit des Geistes and durch ihn hindürch die Wirklichkeit überhaupt. - Die kritische Methode nicht, weil es ihr unter keinen Umständen möglich ist, über die Formen der Gegebenheit hinauszukommen zu dem Gegebenen selbst. Die intuitive Methode nicht, weil sie über der Versenkung in jedes mögliche Gegebene die Form der Gegebenheit iiberhaupt anßer Acht lassen muß. Die kritische Methode kommt nicht zam "Was" der Dinge, die intui-

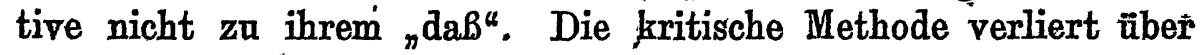
dem Problem der Realität die Realität selbst. Sie wird Formalismus; die intuitive verliert über der Anschauung deś Wirklichen das Problem der Realität; sie wird Romantik und Reaktion. Das Problem des Unbedingten aber ist der Punkt, wo der Unterschied von Existenz and Wesen aufgehoben und damit das Nebeneinander der Methode unmöglich ist. Hier ist gebieterisch eine Methode gefordert; in der beide eins sind; "kritisch-intuiti " " das isst èine Forderung; and wenn sie ganz erfüllt ist, wird auch der adäquate Name geboren sein. Es scheint mir àber in Folgendem ihr Wesen zu bestehen: Sie ruht auf dem Boden der kritischen Methode; sie geht aus vọn den Funktionen des Geistes als den Formen aller Gegebenheit. Aber sie wendet sich auf sich selbst zarück and sieht, dāh alle diese Formen mèhr als leere Formen nur dadurch sind, daß sie erfüllt siñ mit dem Gehàlt eines Unbedingt-Wirklichen, das jeder Einzelform, wie der Totalität aller Formen unerfaBbar ist. Das in Allem . Sinngebende ist nicht selbst ein Sinn, auch nicht die Gesamtheit, auch nicht die Unendlichkeit des Sinnes; 
Die Überwindung des Religionsibegriffs in der Religionsphilosophie. 467

das in Allem Reale ist nicht selbst ein Reales, anch nicht die Gesamtheit, auch nicht die Unendlichkeit des Realen. Das za sehen aber ist nicht mehr Sache der Kritik, sondern der Intaition; wo die Kritik ihre Grenzbegriffe, đ. h. die Dokamente ihrer Begrenztheit setzt, da schaut die Intaition das Unbedingt-Wirkliche, das freilich für sie nicht jenseits der Grenzpfähle, sonderñ mitten im Lande der Kritik die Realitätswarzel darbietet, von der alle Kritik lebt. Es 'ist die Methode des Paradox, der ständigen Durchbrechang und Aufhebung der Form za Gansten des Wirlilichen' in ihr. Nicht Formlosigkeit, nicht fremde Formherrschaft darf die kritische Form durchbrechen; das wäre Verzicht auf Methode, d. h. anf Philosophie; sondern bei vollem Ja zur autonomen, kritischen Form soll der Gehalt des Unbedingten hervorbrechen and zerbrechen, nicht formlos, sondern paradox. Leben in dieser höchsten Spannung ist Leben aus Gott. Anschauen dieser unendlichen Paradoxie ist Denken über Gott, and wenn es methodisch wird, Religionsphilosophie oder Theologie. Niemand freilich kann methodisch za dieser Methode gezwungen werden, wie er zur bloß kritischen Methode gezwangen werden kann; es ist möglich zu leben and zu denken, ohne die Wurzel za sehen, aus der man lebt and denkt, es ist möglich, das Unbedingte zum Grenzbegriff, zum Idealbegriff and dgl. za machen, es in die Peripherie za schieben und in der Autonomie der bloßen Form zu bleiben. Es ist möglich, aber es ist in seinen Konsequenzen Selbstzerstörung, und das führt zum zweiten, der Geschichtsphilosophie.

2. Theonom möchte ich eine Geisteslage nönnen, in weleher alle Formen des geistigen Lebens Ausdruck des in ihnen darchbrechenden Unbedingt-Wirklichen sind. Es sind Formen, also Gesetze, vónor darum theonom. Aber es sind Formen, deren Sinn nicht in ihnen selbst liegt, es sind Gesetze, die das alles Gesetz Darchbrechende fassen; darum the o nom. In gewissen Pexioden z. B. des abendländischen Mittelalters war diese Geisteslage annähernd verwirklicht. Sobald eine Periode der Theonomie ihrem Ende zugeht, sucht sie die Formen, die einmal der adäquate Ausdruck ihres Gehaltes waren, zu konservieren; diese Formen aber sind leer geworden; werden sie mit Gewalt aufrecht erhalten, so entsteht Heteronomie. Heteronomie geht immer von der Religion aus, die Gott verloren hat, die blobe Religion geworden ist. ' Im Gegensatz zn Heteronomie wächst die Autonomie. Autonomie ist immer der Rückschlag gegen die Autonomie der bloBen Religion, 
die alle Kultar anter ihre Heteronomie bringen will. Antonomie der Religion gegen Gott schafft Autonomie der Kaltar gegen die Religion. Der Ansgang des Mittelalters ist typisch für diese Geisteslage. Die autonome Kultur ist im Recht gegen die Religion; es ist das Recht der logischen Form gegen eine ehemals paradoxe, dann ihres Sinnes beranbte Form, die nun als einfacher Widersinn das Logische vergewaltigen will. Hier ist der Sieg der autonomen Form, im Logischen wie im Ästhetischen, im Rechtlichen wie im Ethischen von vornherein entschieden. Und dieser Sieg bedeutet Einsicht in die gegenständlichen Formen der Dinge, bedentet exakte Wissenschaft, bedeutet technisch-rationale Weltbeherrschang.

Aber der Sieg ist teuer erkauft. Das Recht der Antonomie gegenüber der Heteronomie wird zum Unrecht gegenüber der Theonomie, denn die antonome Form ist Gesetz. Mit dem Gesetz kann man technisieren und rationalisieren, aber unter dem Gesetz kann man nicht leben.. Wo das Unbedingte in keiner anderen Weise erfabt wird, als. in der unbedingten Geltuing der logischen oder ethischen oder ästhetischen Form, da tötet es das Leben; denñ da ist es der Richter, der jede einzelne Form verurteilt, weil sie das Gesetz nicht erfüllt, weil sie die Bedingtheit des-Unbedingten nicht erreicht. Därum maß jede autonome Periode zerbrechen: Sie kann mit ihrer formalen Unbedingtheit alles Lebendige töten and rationalisieren; aber sie kann nicht einen einzigen Lebensinhalt schaffen. Sie verliert die Wahrheit und bleibt in der leeren Form der Identitätt, sie verliert die Persönlichkeit und bleibt in der leeren Form des ;Da sollst". Sie verliert die Schönheịt nnd bleibt in der leeren Form der Synthesis : sie verliert die Gemeinschaft und bleibt in der leeren Form der Glejichheit. Alles verzweifelte Ringen aber um die Erfüllng dieser Formen im Logischen wie im Ethischen, im Denken, wie im Handeln ist nur der . Ausdruck für die Tragik der Aatonomie.

Dieses Ringen ist von überwältigender Größe und diese Tragik von erschütternder Tiefe. . Es sind die Zeiten der großen individuellen Kalturschöpfungeñ"; aber das Ende ist' das Schwanken z̀wischen ansprachsv́ollem Rationalismus ünd verzweifelndẹr Skepsis im Logischen, und zwischen Pharìisäismus and Gesetzlosigkeit im Ëthischen: Die Aatonomie bricht anseinander in Nomismus und Antinomismaș. Lebensfähig bleiben nar diejenigen, welche sich den großen Spannangen des Geistes entziehen and die autonome 
Form benutzen zu Technik and Taktik in Wissenschaft und Wirtschaft, in Politik und Kunst. Sie haben ihren Lohn dahin. Der Lohn aber des Geistes, der aúsharrt, ist das Darchbrechen des Unbedingten darch alle Formen, nicht als Gesetz, sondern als Gnade, als Schiksal, als unmittelbare überwältigende Wirklichkeit. - wie es z. B. der Antike beschieden war, in der Dóppelfórm der neuplatonischen Mystik auf logischem. und̆ des Christentums auf ethischem Boden.

Das Thema der Geistesgeschichte ișt der Kampf von Theonomie and Aatonomie. Die Theonomie ist sieghaft, solange sie lebendiger Durchbruch ist, solange die Paradoxie als Paradoxie erlebt wird. Sie ist aber dem Verhängnis verfallen, immer wieder ans dem lebendigen Paradox einen objektiven Widerspruch machen zu müssen; dann steigt ans dem Kampf gegen ihre Heteroñomite die Autonomie der Form sieghaft empor, tum schlieblich ihrem eignen Verhängnis, der Auflösung entgegenzugehen. Das ist.nicht bloB im Nacheinander gemeint. In jedem Angenblick der Geistesgeschichte tobt dieser Kampf. Aber der Sieg und die Niederlage des einen oder anderen gibt auch ein Nacheinander, eine Geschichtsphilosophie nicht nur des Querschnitts, sondern auch des Längsschnitts.

Wir haben das Ringen beider in der Religionsphilosophio betrachtet; sie ist der Ort, wo der Kampf am deutlichsten sichtbar ist. Sie ist selbst in ihrer Entwicklung ein Teil dieses Kampfes. Nar weil ihr die autonome. Entwicklung die Formen gegeben hat, kann sie Philosophie sein; nar wo ihr die Theonomie den Gehalt, die - Wurzelung im Unbedingten gibt, kann sie Religions-Philosophie sein. Sie kann es aber nar, wenn sie sich der Herrschaft des Begriffs entzieht, der das typische Symbol der antonomen, Gott abgewandten Periode ist, des Begriffs der Religion, wenn sie einsieht, daB nicht die Religion der Anfang and das Ende and die Mitte in allem ist, sondern Gott, and daB jede Religion and jede Religionsphilosophie Gott verlieren, wenn sie sich nicht auf den Boden des Wortes stellen: Impossibile est, sine deo discere deum. Gott wird nur erkannt aus Gott. 